\title{
More-Than Human Centred Design: Considering Other Things
}

This paper responds to contemporary design contexts that frequently contain complex interdependencies of human and non-human actants. To adequately represent these perspectives requires a shift towards More-Than Human Centred Design. The Internet of Things is one context that demonstrates this need. The 'things' within such networks transcend their physical forms and extend to include algorithms, humans, data, business models, etc., and each imports independent-but-interdependent motivations and perspectives. Therefore, we use the Internet of Things to clarify our proposition and to convey our three contributions. First, we review the expanding corpus of contemporary HumanComputer Interaction research that seeks to expand the notion of Human Centred Design by moving beyond the dominant anthropocentric perspective. Second, we introduce a novel design metaphor, 'constellations', which allows both the interdependencies and independent perspectives to be considered. Third, we provide an account of a speculative design to demonstrate how it may be put into practice.

Keywords: Internet of Things; Non-anthropocentric; Object Oriented Ontology, Speculative Design, Design Fiction.

\section{Introduction}

In The Design of Everyday Things (2013), Don Norman presented principles for designing 'things' in such a way that human interaction with them is smooth and fruitful or 'Human-Centred'. Until recently, these interactions occurred almost exclusively between users and things within independent standalone systems. Norman cites numerous examples including a refrigerator, a telephone, and a clock. Although some of these examples, i.e. the telephone, incorporate several technologies interacting across multiple layers, the experience of using them is encapsulated within a discrete interface.

In this paper, we question whether the self-contained interactions, from which Norman's insights emerged, are still representative of our current interactions with technology. The question arises because it has become more prevalent for interactions 
to occur as part of, and with impact upon, increasingly complex network ecologies. Exemplifying this, our interactions with so-called Internet of Things (IoT) devices often appear familiar, yet, invariably, they produce an unseen digital residue that facilitate other forms of interaction with or for additional unseen actants. What differentiates IoT devices from their non-IoT counterparts is not simply the added complexity of these systems but rather the new types of agency, value, and power that they enable through the 'networkification of the existing non-Internet world' (Pierce and DiSalvo 2017).

In stark contrast to the 'visibility, appropriate clues, and feedback of one's actions' that Norman highlights as key properties of human-centeredness (Norman 2013,8-9), the digital footprint of modern IoT devices is rarely clear to the humans whose activity underpins the creation of this data-rich residue. The relationship between the interfaces, users, use, and residual data, in some cases, problematizes some of the core ideals of Human Centred Design (HCD) because at the point of interaction, significant portions of an IoT device's activities and the interdependencies with other actants are often obfuscated to an extent that it undermines the agency of the human user.

In the following, we elaborate on this further by discussing HCD, the IoT, and the difficulties arising when one meets the other.

\section{What do we mean by Human Centred Design anyway?}

Whilst prefixing More-Than (to HCD) in the paper's title is supposed to infer some critique of HCD, this does not extend to the entirety of what HCD represents or encompasses, nor what we perceive to be the underlying and laudable intentions of most HCD-informed projects. Hence, the following section clarifies the nature of the critique.

HCD has its roots in computer science and ergonomics as exemplified within the associated international standard (ISO 9241-210). The standard aims to underpin 
management strategies for large technical projects, describing how to provision hardware and software that enhance human-system interactions. Although demonstrably useful in certain circumstances, this engineering-focused stance has a tendency to assert predetermined functions and assumptions about design contexts (Giacomin 2014) which results in a somewhat reductive representation of 'the human'. Contrastingly, the widely accepted notion of 'situatedness' insists that "the coherence of action is not adequately explained by either preconceived cognitive schema or institutionalized social norms" (Suchman 2017,177) that are prevalent within engineering-focussed HCD.

\section{Beyond Usability}

Moving beyond the relative-straightforwardness of usability evaluations, more contemporary HCD attempts to amalgamate insights garnered from potential future users to transcend those which the individual users themselves might have realised (Giacomin 2014). However, balancing professional intuition of a designer or researcher against the situated knowledge and desires of users remains a substantive challenge for those applying HCD methods (Steen 2011). If these difficulties are correctly mediated then the resulting human-centred designs should be "physically, perceptually, cognitively and emotionally intuitive' (Giacomin 2014) whilst matching 'the needs and capabilities of the people for whom they are intended' (Norman 2013,9). HCD tools are then often used to assess whether a design successfully integrates these attributes (Greenberg and Buxton 2008) although there are numerous problematic examples that testify that 'there is no simple recipe for the design or use of human-centred computing' (Kling et al. 1997). 


\section{Transcending Simplicity}

The pervasiveness and longevity of HCD is a reflection of how it has been successfully leveraged to help design devices, services and software that are efficient, effortless, and edifying to use. A key factor in how HCD achieves this rests in its aim of reducing complexity (or conversely as it is oft-interpreted, increasing simplicity). Simplicity, in HCD terms, suggests that the artefact being designed should fade into the background and become invisible. Any complexity that remains should be that of the underlying task and not of the tool designed to help achieve the task (Norman 1997,50). Although HCD's call for simplicity is well reasoned and, in the right circumstances, can produce desirable outcomes, it is also true that if simplicity is treated dogmatically it can import risk into design processes. Perhaps unsurprisingly this blunt interpretation of simplicity constrains HCD approaches to a 'limited view of design' and results in analyses preoccupied with 'page-by-page' and 'screen-by-screen' evaluations (Norman 2005), distracted by minutiae and devoid of contextual awareness. Meaningfully, handling simplicity is increasingly problematic in relation to the societal, economic and environmental challenges societies now face. This is evident within the context of the hyper-connected and data-mediated assemblages that make up the IoT. In particular, designs that interpret HCD's simplicity axiom to mean that maximising simplicity (of interface, interaction, and user experience design) is always the best thing, have a contradictory relationship with HCD because delivering simplicity so bluntly often disenfranchises the user.

\section{Human-Centeredness in HCI}

Much of the design expertise that technology developers have acquired comes from the field of Human-Computer Interaction (HCI), hence it is important to consider HCI's relationship with HCD. HCI has matured from a starting position where it 
developed and evaluated computer systems in terms of their ease of use to a field that frequently tries to ascertain what things users actually need before designing them (Bannon 2011). Increasingly, HCI professionals are asked to 'Look at this area of life and find us something interesting' (ibid). This evolution roughly maps to what is described as HCI's three 'waves' (Bodker 2006). The first wave was defined by a model-driven approach to understanding usability. Second-wave HCI embraced humancentred techniques but was still blinkered by a preoccupation with trying to discretely describe unquantifiable notions, such as, 'efficiency' and 'appropriateness'. Third wave approaches appreciate the pervasiveness of technology across home and work environments together, and, as such, acknowledge the need to deal in nebulous, and emergent constructs. This more complex epistemic landscape necessitates what Bødker calls 'artifact ecologies' (2015) to represent the emergence of technology use over time, in mixed contexts, and among a variety of devices. These third wave design challenges resonate with those we encounter within the IoT. When applied in these complex contexts and viewed dogmatically, HCD approaches reveal themselves as 'an incomplete philosophy that lacks a sense of responsibility for concerns other than those of the immediate end user' (Schweikardt 2009).

These third wave design contexts, including the IoT, are network ecologies. The data which enable and drive them, punctuate and texture their complexities whilst the interdependent-but-independent attributes of their constituents confound the incompleteness of HCD's foundation. Therefore, if HCD is the prevalent design philosophy of the day, then it is crucial that the HCD design tools are able to reflect the nature of these ecologies. This, however, is not straightforward; in a networked world with many connected actants — both human and non-human — the notion of human- 
centred ceases to be useful. When designing for networked ecologies prescribing a unique centre cannot properly characterise the design space.

\section{We Need to Talk About the IoT}

Returning to one of Norman's HCD examples, the wired telephone, although part of a complex global network from the user's perspective is very simple: when it rings pick it up and say "Hello" or, to make a call, pick it up and dial the relevant number. Smartphone interfaces, designed utilising HCD methods, operate similarly in terms of how users make or receive calls, and thus this simple human-centred interaction pattern seems transferrable. However, looking deeper into the situation quickly reveals more nuance. The wired phone is a single purpose device, connecting into the phone network, which itself is a complex but highly sanitized and controlled system. The smartphone on the other hand is a general-purpose computer, imbued with a range of sensors, connected to the Internet, running user-installed software. It so happens that it also makes phone calls and, for historical reasons, is primarily referred to as a 'phone'. This is analogous to the contrast between 'things' and the IoT. Imagine a door lock. A non-IoT door lock is mechanical and serves a single purpose, but it's IoT counterpart is digitally-operated and generates data, it participates in an interrogatable global network. By obfuscating the role that data plays in defining IoT devices, we contend that simplicity-focused HCD can demonstrably result in the design of user interfaces and devices which de-privilege the user, and hence are arguably counter to HCD's purported ideology. In the following we describe various IoT devices which exhibit design that by some measures are 'Human Centred', but that also demonstrate unseen agendas, unclear agency, and inconsistent design choices that conspire to undermine the needs of their users. 
The manufacturer Vizio sold smart televisions that logged users' activity and passed this data to third parties for marketing purposes. While this practice is common in the smart TV industry, Vizio failed to include details of this in the user agreement and hence the gathering and sale of this data was illegal. Illegality aside, the relevant point, vis-à-vis HCD, is that data logged and distributed via these televisions had no relevance or impact on the user's activity (watching television). Rather than encapsulating the complexity of the task in a simple-to-use tool extends the task to do something that most users probably do not desire. Manufacturers defend the gathering of data like this, claiming that such collection helps improve user experience. However, the issue is not what the data is used for, it is that the interactions necessary to operate the TV do not, in any obvious way, show or represent that the data generated from those interactions is being harvested (or subsequently sold). The dilemma for HCD lies between the observation that obscuring the role of data gathering clearly diminishes user agency, it is also true that revealing this part of the IoT system would arguably add to the complexity of 'watching television'.

Another area that also problematizes HCD's desire for simplicity is device or platform security. In 2016 the 'Mirai' Botnet attack disabled the Domain Name Servers of internet provider Dyn, resulting in disruption to many services, including Netflix, Twitter, and Airbnb. The attack utilized hundreds of thousands of enslaved IoT devices. Many of these devices were compromised because they were not firewalled, had insecure passwords, and/or their owners/administrators had not changed the insecureby-default configuration. Whilst administrative ineptitude and lack of security auditing partly explains the exposure of this straightforward attack vector, the desire to design devices with the lowest possible barrier to usability is a contributory factor we should not ignore. In creating devices that were simple to setup and configure, designers 
removed the need for users to engage with their device's administration. This is, perhaps, the most elementary demonstration of how efforts to simplify user experience — possibly to fulfil a HCD requirement—can have serious ramifications (Realpe-Muñoz et al. 2016).

Additionally, the architectures of IoT device infrastructures can also cause problems for users. Many IoT devices are supported by or reliant upon cloud services. These provide data storage and data processing to a level which would not be feasible at the 'edge' or on the IoT devices themselves. While often useful, and occasionally necessary, these architectures create interdependencies. Such interdependence has oft resulted in devices becoming unreliable or obsolete when/if the cloud service becomes temporarily unavailable or permanently discontinued. Notable examples of cloud support being withdrawn from devices include Nest's Revolv hub, Pebble Smartwatches, and Nabaztag rabbits. Consonant with the prior examples, HCD approaches may lead toward designs whose cloud-dependency can result in functions and simplicity that would not otherwise be possible, yet in some circumstances this may prove counterproductive to HCD ideals.

These examples cannot be considered representative of the entirety of HCD practice, nor do we proffer it as a universal critique. Rather, we present them to illustrate issues that may arguably be correlated to aspects of HCD and hence that didactically assuming that enhanced simplicity produces designs that are more humancentred, is a questionable viewpoint to hold.

\section{Taking A Non-Anthropocentric Turn}

We now consider a series of theoretical responses to these emerging complex network ecologies that are united in their Non-Anthropocentric stances. 
Reflecting on the $21^{\text {st }}$ century's 'product-scape' Cila et al (2017) discuss modern products and their ability to network with each other, collect data via sensors, and perform computation. They adopt the metaphor of agency to illuminate how designbased inquiries tend to lack the ability to explore the broader societal impacts of these IoT devices, and cast 'things' as socio-material assemblies (ibid). Their goal of identifying 'methods of design research that give both [human and nonhuman] an equal voice' is driven by their desire to expose the unique properties of IoT devices. The paper's argument is founded on a non-exclusive consideration of theoretical positions including Actor Network Theory (ANT) and Object Oriented Ontology (OOO), and suggests approaches for designing and researching IoT devices (ibid).

Chang, et al. (2015) draw on ANT and OOO and New Materialist positions before invoking Animism (the notion that objects exhibit autonomy, inner life, and personality) to create an ethnographic metaphor for eliciting 'thing centred' perspectives - i.e. conveying insights from the point of view of a designed thing, in their case, scooters. This novel approach (which involved video recording a scooter-eye view of Taipei before having those recordings interpreted and then embodied by an actor) aims to 'construct an IoT ecosystem where people and things [...] appropriately collaborate [so that] the relationship and social meanings between things and people should be more carefully considered' (ibid).

Motivated to explore the gap between things and humans in IoT contexts, Morse Things (Wakkary et al. 2017) involved the design of a set of cups and bowls that, although connected to the Internet, only ever communicate with each other by Morse code. The work conceptualises domestic objects that are neither human-centred, nor thing-centred, cultivating a new and 'special relationship that spurs ongoing reflections and interpretations despite knowing these can never be resolved' (ibid). The research is 
partly methodological, but also makes the case for new 'vocabularies, practices, and methods' to meaningfully explore the gap between things and humans in support of a 'thing-centred IoT and interaction design agenda' (ibid).

In discussing Post Userism, Baumer and Brubaker (2017) carefully consider the user construct as it manifests in design research. They note how, within the broadening scope of design research, notions of 'user' can result in reductive representations when confounded by increasingly blurred boundaries between human and computer, and casts significant doubt on what the 'centre' of design research should be. They use 'subject positions' to explain how accounting for the relationships within a system may provide designers with ways of moving "beyond the traditional bounds of "user"' (ibid), not to solve historical problems, but 'to support such conversations about the centre around which our discipline is organized' (ibid).

Notwithstanding the wealth of other recent-and-related research, our endeavour is to identify commonalities and gaps between these positions, and articulate to what end we can reflect on the common aspects while aspiring to also fill in the gaps.

\section{Minding The Gap}

Within the aforementioned research there is significant shared theoretical ground. In particular ANT and OOO are referred to across the aforementioned works, whilst more niche, but similarly relatable, concepts such as Animism and PostPhenomenology also make occasional appearances. Further commonalities include these research projects being driven by the need to address the new complexities which arise from our increasingly interdependent cohabitation with the IoT, and their conclusions leaning towards the call to develop new methods for helping designers meaningfully respond to this emerging landscape. Collectively these examples illustrate 
a consensus forming around the idea that non-anthropocentric theories have increasing relevance for contemporary design.

Although rendered from disparate philosophical foundations the direct impact of these various theories on practical design work is, relatively speaking, negligible. Summing up how their 'epistemological commitments' turned out, Wakkary, et al. say, 'Our insights reflect and engage [OOO and post-phenomenology] through design' but do not delve deeper (2017), while Cila, et al. conclude, 'we do not adopt the arguments of any of these approaches directly as our theoretical foundation but rather aim to appropriate their main message' (2017). Hence, while a relationship with theory is often important to these design research projects, the precise nature of that relationship often appears not to be of direct consequence to the outcomes. Irrespective of any given theory's nuance and lingua franca, once a design is made tangible, whatever theory helped inform a design, the pendulum of relevance swings squarely towards the designed thing itself, rather than the theory that was in mind when it was conceived.

Seen together, we might say that these related non-anthropocentric design approaches constitute a broader movement, albeit one that is, as yet, 'pre-paradigmatic', i.e. with 'competition amongst a number of different schools of thought, usually drawing on different philosophical foundations' (Gaver 2012). While such preparadigmatic competition is ultimately productive, in the present it can also be stifling and cause individuals to become distracted by the need to justify the foundations rather than trusting it will be valued and understood by their peers (ibid). The haziness emanating from the pre-paradigmatic nature of the movement is another plausible reason why, within the reviewed literature, there are several occasions where the authors invoke multiple theoretical perspectives without an explicit explanation for why one, rather than another, was ultimately the theory with which they worked. 
In summation, there seems to be a common motivation among many researchers, and this composes a non-anthropocentric design movement. Network anxieties resulting from pervasive socio-technical ecologies, like the IoT, have encouraged researchers to consider non-anthropocentric theories to inform their inquiries. Driving these underlying anxieties is the problematic nature of HCD's limitations that emerge when utilised in complex or third wave contexts such as the IoT. In the space between these considerations there is an opportunity to develop a paradigmatic fulcrum around which HCD can be augmented with new metaphors that reflect the motivations for, and the detail of, the theories which contribute to this non-anthropocentric turn.

\section{Towards More-Than Human Centred Design}

Thus far, we have argued and identified an opportunity to augment the collection of methods referred to as HCD by incorporating non-anthropocentric theories to enable a multiplicity of perspectives that are more apt to be used among the emergent complexities of modern socio-technical design contexts like the IoT. Although it is a significant undertaking, what follows is one proposal for how we might start to do this by introducing a reusable design metaphor constellations which aims to harmonise the disparate non-anthropocentric theories accessibly. This may help reframe design such that HCD better reflects the complex interdependencies of human and non-human actants and the independence of contextual perspectives, while maintaining the underlying ideals of HCD. In essence, our aim is to 'blackbox' (Latour 1999) the sometimes-unwieldy philosophy, and encapsulate it into an approachable construct. Although our aim is to free design practitioners from being overly concerned with the nuances of individual non-anthropocentric theories, our proposal for the constellation metaphor is, inevitably, couched in one of them—Object Oriented Ontology. 


\section{Object Oriented Ontology}

While all of the non-anthropocentric positions discussed have their merits, for our purposes OOO has distinct advantages. Object Oriented Ontology is a study of being that revolves around so-called 'objects'. While there is much debate about the nuance of how this plays out (Harman 2015), for our purposes an object is any selfcontained construct. For example, a conference, a paper, a word, a citation, a committee chairperson, and an arm chair, physical things, conceptual things, even a theory itself (Lindley et al 2017) — all are 'objects' in terms of OOO. Because of this inherent, if rather strange, ontological property of $\mathrm{OOO}$, it is reasonable to assert that if we were to imagine a taxonomy of non-anthropocentric theories, because all the other theories can resolve to an OOO-object, OOO itself would sit 'high-up' within the taxonomy. Put differently, while it is relatively straightforward to explain any theory as an OOO object without undermining either position, the inverse is less practicable (e.g. to cast OOO as an 'actor' in ANT terms).

The extreme flexibility that OOO's view of the world lends to the theory that it is founded upon a rejection of 'correlationism' (the view that 'things' are only real insofar as they are perceived by human subjects, which in turn is the result of a correlation between 'what it is to think' and 'what it is to be'). By rejecting this position OOO seeks to acknowledge and appreciate the 'tiny ontologies' (Bogost 2012,19-22) of all objects. Although tiny ontologies are often incompatible with each other (i.e. it is hard for the comfy arm chair to make sense of the hard conference chair's world because they don't have any shared ground), in spite of this, OOO contends that one object should not be privileged more than another object, whatever they may be, and however incompatible.

Extending the more familiar Heideggerian position that 'things' (objects) make sense in terms of their utility or purpose, OOO takes the idea further by suggesting that 
objects are not defined by their 'readiness at hand' for humans, but for any given object. Hence, any object's notion of being and reality is constructed on unique and ultimately particular terms (DiSalvo and Lukens 2011). Given the ontology of any given object is completely particular, and mostly imperceptible to human objects (Harman 2011, 2), the relationship between them are rarely intimate. With all this said, our encounters do become more cosy when the objects in question are technologies that, like IoT devices, have been designed specifically to act and have agency in our world (Bryant 2014).

Much of what we want to achieve with this paper proposition hinges on OOO's broad scope, flexibility, and the potential to be reflective of other theories without tarnishing either one's essence. In the following we build upon an OOO foundation to construct and advocate for a new metaphor-constellations - which is intended to turn OOO into an accessible form for application in design/research projects and that may incorporate elements of other related non-anthropocentric positions.

\section{Constellations}

Pierce and DiSalvo rather poetically sum up why a metaphor could be a fruitful way to approach designing for the IoT; 'Shapeless and faceless, everywhere and nowhere $[\ldots]$ the Internet must be grasped in metaphorical terms' (2017). Employing design metaphors is certainly not a new idea (Schön 1993) but the complexities of our networked world seem ripe for new metaphors. As Cila et al. put it, 'metaphors vividly evoke the invisible, yet powerfully present potential of [the] IoT' (2017). We propose constellations as a metaphorical way to frame OOO-thinking for designers working in contexts like the IoT.

Walter Benjamin describes how the meaning of any collection of things varies wildly dependent on the observer's perspective, noting 'ideas are to objects as constellations are to stars' (1999). This particularity of meaning dependent on 
perspective naturally resonates with the OOO thesis and led us toward developing the constellation metaphor for considering contexts like the IoT. In short, this metaphor represents the idea that, as with the cosmological constellations in the night sky and their constituent stars, IoT things are simultaneously 'stars' in their own right, as well as being part of groups, or constellations. Depending on what perspective an observer takes, how these constellations appear varies wildly. Similarly, while IoT devices exist individually, their meaning and significance is augmented by virtue of being part of a wider constellation. These constellations are objects in their own right, specifically they are an object that can transcend the physicality of the IoT device itself, and represent or appreciate intangibles like business models, algorithms, or regulations.

While one's physical position quite clearly effects how a constellation appears, Benjamin's suggestion that things can appear quite differently goes beyond just geometry. Different cultures observing the same constellations of stars interpret them variably too: The Big Dipper, The Plough, The Stretcher, and Wise Men with Drinking Gourd - all refer to the same collection of stars but reflect environmentally dependent cultural constructions of what those stars mean. Hence, from differing appearances based on geometry, combined with varying environmental and cultural prejudices, vastly different interpretations on what are the same underlying entities, emerge. Taking this to the extreme, individual parts of the constellation can entirely disappear from the view (of some observers) but without necessarily diminishing their relevance or influence. For example, on Earth, for observation points south of the equator, the star Polaris disappears entirely. Importantly, however, simply because Polaris is not always visible to everyone on Earth does not mean it ceases to exist or is less significant. Likewise, just because one cannot see an IoT provider's business model, does not mean 
that this model will not have a significant impact on how the product works, on what data it generates or processes, and what that assemblage means.

To concretize this metaphor for the IoT, consider a domestic IoT-enabled heating system. Multiple entities must come together to make this system work including the IoT device itself (e.g. a smart thermostat); a supporting cloud service; control software; the central heating/cooling system that is being controlled; the business model of the provider; the algorithms which optimize the system. Applying the constellation metaphor, whilst bearing in mind OOO's lessons, we might want to consider the geometric and cultural factors impacting each of the perspectives of each 'star' in the constellation. For example, a user cannot see the algorithms that power the system or the business model that supports the ongoing viability of the cloud service, however they may well feel their effects. In contrast the user directly interacts with the IoT device, but arguably from the IoT device's point of view, the digital relationship with the cloud server and mechanical relationship with the heating/cooling system is more consequential. Employing this metaphor sidesteps the problematic notion of 'centre' and leaves behind a more appropriate flexible framing that can appreciate and respond to both independence and interdependence.

We acknowledge that the expansiveness of the metaphor is potentially challenging but suggest that in practice context-specific perspectives would become the focus, and thus not deplete the usefulness of the metaphor. Challenges notwithstanding, it seems the extensibility of the constellation metaphor's scope is also a key strength; it can be stretched or shrunk to include the objects that are of relevance for any given context or situation. In design terms, if the house from our IoT-heating example was temporarily occupied by an Airbnb customer then their temporary occupancy would have a direct impact on the shape and scope of the constellations. As a guest, they 
would have a different perspective on the constellation compared to the homeowner. Also, although invisible to each other, the customer's presence in the house would have an impact on the algorithms' view of the house, and vice versa. The bounds of the constellation(s) should be adjusted as and when necessary to, either generatively or critically, examine different perspectives. In this discussion, the mechanics of using the constellation metaphor are deliberately kept simple and open-ended, but the key issue is that while it is not routinely necessary (and arguably possible) to conceive of all possible perspectives all of the time, it is crucial to accept that they exist and consider which are of relevance for any given creative or analytical task.

\section{Designing with Constellations}

In this section we demonstrate how designers may use constellations in practice. It is important to note that the design work presented, our engagement with $\mathrm{OOO}$, and the constellation metaphor itself, co-evolved together. Hence, the metaphor has emerged from the designs as much as the designs have emerged from the metaphor; as such, the work represents a Research through Design project (Gaver 2012). Articulating how a metaphor impacts on design work, as with articulating what it is to 'do design', is more of an art than a science. Hence, though we cannot didactically tell the reader how the constellation metaphor 'should' be applied in terms of a step-by-step method, the following commentary is intended to put flesh on the skeleton described thus far and give one example of how it could be utilised. The work described below explores using the constellation metaphor in the creation of a Design Fiction project, which in particular aligns with the 'Design Fiction as World Building' approach (Coulton et al. 2017). 


\section{Orbit: Designing for Bespoke Dynamic Privacy Settings}

This work focused on exploring 'Implications for Adoption' (Lindley et al. 2017a) relating to IoT products in the home, against the backdrop of the EU's General Data Protection Regulations (GDPR). Under GDPR, when consent is given to collect personal data, the burden lies with the 'data controller' (i.e. technology company) to verifiably show that the consent was gained freely, specifically, and unambiguously. With this in mind, we were interested in researching the interactions that facilitate the gaining of consent and, in any given context, the associated impact on relevant parts of the constellation.

As a Design Fiction World Building process, we began by selecting a specific IoT device to construct the particulars of the fictional world around, in this case an IoT door lock. We endowed the fictional IoT lock with four possible ways of being operated with various requirements on users' data: Using a smartphone as the key to unlock the door (by utilising near-field communication this can be achieved while all data is selfcontained on the user's own devices); geofencing to automatically lock the door when a user leaves the house (data must be shared with the cloud service of the lock); voice activated unlocking via integration with Amazon Echo (data must be shared with the lock company's cloud and Amazon's cloud); extensible integrations via a service such as 'IFTTT' (data must be shared with the lock company, with IFTTT, and potentially with multiple unknown $3^{\text {rd }}$ parties). Within this diegesis to gain unambiguous consent in each configuration, the installation of the lock system required the design of privacymanagement software called Orbit. Exploring the lock's constellations formed a key part of the contextual research for the design of Orbit.

Studying existing IoT devices in order to extrapolate how the lock's different functionality may impact on how data are used, showed that once several data controllers are implicated, the ambiguity in the forms-of-words within user licence 
agreements and privacy policies make it almost impossible to know what data is collected and where and what they may be used for at any given time. Constellation thinking provided a means to unpack and apprehend this uncertainty by adopting the perspectives of the interdependent actants within the constellation. Acknowledging the multiple constituents of the constellation, Figure 1 maps flows of data resulting from the lock's most sophisticated feature set being utilised to turn on lights when the lock is activated. Creating and considering such maps forces designers to explore each actant's perspective of the design case (i.e. the lock) but also on every other actant within the constellation.

In response to, and provoked by, our consideration of the constellation, the design brief led us to consider notions such as "what data that could be used to identify the user'. Whilst anonymity may be a preference for the user, gathering, holding and processing data that could identify the user was not only preferable but also necessary for some other constituents in the constellation to perform properly. Balancing this dilemma of multiple contrasting needs against the our primary concern being the desires and experience of the human end-user, the constellation provided a unique lens with which to focus the problem through. In lieu of any certainty about precisely what data might be shared, when and with whom, this led us to explore 'likelihood' rather than absolutes. This is reflective of the fact that whilst data at the point of interaction can be quantified, where it flows, to whom, and what is done with it, is dependent on many actant-specific factors such as regulatory environment, standards for interoperability, the prevailing market forces at the time, and how it might ultimately be combined with other data. Notwithstanding the irony that in search of a means to gain 'unambiguous consent' it seemed necessary to represent ambiguity within the interface, the resulting design is shown in Figure 2. 
The design includes three data levels, or 'orbits', which are arranged as concentric circles where the inner circle codifies data that is controlled solely by the user; the middle circle refers to data held by known providers (e.g. the lock company); while the outer orbit represents unknown $3^{\text {rd }}$ parties (in our example IFTTT and any other parties' data is exchanged with). The boundaries between the orbits' boundaries can be blurred or sharpened in order to represent certainty. Hence, using these orbits it is possible to show identifiability for any given configuration, including those which do not have a discrete outcome because of the constellation's intricacies. In order to explore how this concept would work in situ, we placed the concept design into the diegesis of our Design Fiction. An 'entry point' into the Design Fiction, a video which demonstrates how the concept could be used to generate a bespoke, visually-led, privacy agreement based on user choices as part of the setup of the IoT device. The video shows a user using their voice assistant to 'detect new devices' before being directed to use the Orbit privacy app. Illustrated in Figure 3, a slider allows the user to turn on/off features, while the display updates to show the relevant impact on personal identifiability as per our constellation-informed concept design.

Other applications of the constellation metaphor will clearly appear very differently depending on the context. However, the Orbit example is indicative of constellations' potential to shed light on design challenges that are inseparable from their involvement in heterogeneous IoT networks.

\section{Conclusions}

The paper argues that the interdependent-but-independent perspectives that arise when designing for contexts like the IoT demand that HCD approaches be expanded to become More-Than Human Centred Design. To this end we present the constellation 
metaphor, which we believe is an accessible and useful framing for designers and researchers to utilise non-anthropocentric theory in practice. The proposals in the paper are contingent; we do not intend to describe enduring visions for More-Than Human Centred Design or the constellation metaphor, nor to prescribe with exactitudes how this approach should cohere with other aspects of a HCD design process. Notwithstanding those caveats, based upon our synthesis of theory and practical exploration through Design Fiction, we believe this is a valuable addition to the gamut of HCD approaches.

HCD is undoubtedly a permanent fixture in design, but we must ensure HCD evolves to reflect contemporary contexts, such as the IoT. Constellations may represent an additional complication in the already-complex process of identifying and utilising HCD tools for a given project. However, reflecting and utilising burgeoning nonanthropocentric perspectives within HCD processes is an important and relevant endeavour. Using the constellation metaphor to practice More-Than Human Centred Design is a viable and accessible way to achieve this.

\section{Acknowledgements}

This research was enabled through

\section{References}

Bannon, Liam. 2012 "Reimagining HCI: Toward a more human-centered perspective." interactions 18 , no. 4: 50-57.

Benjamin, Walter. 1999. The Arcades Project, trans. Howard Eiland and Kevin McLaughlin.".

Bødker, Susanne. 2006. "When second wave HCI meets third wave challenges." In Proceedings of the 4th Nordic conference on Human-computer interaction: changing roles, pp. 1-8. ACM. 
Bødker, Susanne. 2015. "Third-wave HCI, 10 years later---participation and sharing." interactions 22, no. 5: 24-31.

Bogost, Ian. 2012. Alien phenomenology, or, what it's like to be a thing. University of Minnesota Press.

Baumer, Eric, and Jed Brubaker. 2017. "Post-userism." In Proceedings of the 2017 CHI Conference on Human Factors in Computing Systems, pp. 6291-6303. ACM.

Bryant, Levi. 2014. Onto-cartography. Edinburgh University Press, 2014.

Chang, Wen-Wei, Elisa Giaccardi, Lin-Lin Chen, and Rung-Huei Liang. 2017.

"Interview with Things: A First-thing Perspective to Understand the Scooter's

Everyday Socio-material Network in Taiwan." In Proceedings of the 2017

Conference on Designing Interactive Systems, pp. 1001-1012. ACM.

Cila, Nazli, Iskander Smit, Elisa Giaccardi, and Ben Kröse. 2017. "Products as Agents:

Metaphors for Designing the Products of the IoT Age." In Proceedings of the 2017 CHI Conference on Human Factors in Computing Systems, pp. 448-459. ACM.

Coulton, Paul, Joseph Lindley, Miriam Sturdee, and Michael Stead. 2017. "Design Fiction as World Building." In Proceedings of the 3rd Biennial Research Through Design Conference, 163-79. Edinburgh, UK. doi:https://doi.org/10.6084/m9.figshare.4746964.v2.

DiSalvo, Carl, and Jonathan Lukens. 2011. "Nonanthropocentrism and the Nonhuman in Design: Possibilities for Designing New Forms of Engagement with and through Technology." In From Social Butterfly to Engaged Citizen, edited by Marcus Foth, Laura Forlano, Christine Satchell, and Martin Gibbs. The MIT Press. doi:10.7551/mitpress/8744.003.0034.

Gaver, William. 2012. "What Should We Expect from Research through Design?" In Proceedings of the 2012 ACM Annual Conference on Human Factors in Computing Systems - CHI '12, 937-46. New York, New York, USA: ACM Press. doi:10.1145/2207676.2208538.

Giacomin, Joseph. 2014. "What Is Human Centred Design?" The Design Journal 17 (4): 606-23. doi:10.2752/175630614X14056185480186.

Greenberg, Saul, and Bill Buxton. 2008. "Usability Evaluation Considered Harmful (Some of the Time)." In Proceeding of the Twenty-Sixth Annual CHI Conference on Human Factors in Computing Systems - CHI '08, 111-20. New York, New York, USA: ACM Press. doi:10.1145/1357054.1357074.

Harman, Graham. 2011. Tool-being: Heidegger and the metaphysics of objects. Open Court. 
Harman, Graham. 2015. “Object-Oriented Ontology.” In The Palgrave Handbook of Posthumanism in Film and Television, edited by Michael Hauskeller, Thomas D Philbeck, and Curtis D Carbonell, 401-9. London: Palgrave Macmillan UK. doi:10.1057/9781137430328_40.

Kling, Rob, and Susan Leigh Star. 1998. "Human Centered Systems in the Perspective of Organizational and Social Informatics." ACM SIGCAS Computers and Society. doi:10.1145/277351.277356.

Latour, Bruno. 1999. Pandora's Hope: Essays on the Reality of Science Studies. Harvard University Press.

Lindley, Joseph, Paul Coulton, and Rachel Cooper. 2017. "Why the Internet of Things Needs Object Orientated Ontology." The Design Journal 20 (sup1): S2846-57. doi:10.1080/14606925.2017.1352796.

Lindley, Joseph, Paul Coulton, and Miriam Sturdee. 2017. "Implications for Adoption." In Proceedings of the 2017 CHI Conference on Human Factors in Computing Systems - CHI '17, 265-77. New York, New York, USA: ACM Press. doi:10.1145/3025453.3025742.

Norman, Donald. 1998. The invisible computer: why good products can fail, the personal computer is so complex, and information appliances are the solution. MIT press, 1998.

Norman, Donald A. 2005. "HCD Harmful? A Clarification." http://www.jnd.org/dn.mss/hcd_harmful_a_clari.html.

Norman, Donald A. 2013. The Design of Everyday Things (Revised and Expanded Edition). Basic Books.

Pierce, James, and Carl DiSalvo. 2017. "Dark Clouds , Io \$ \#! +, and ? [ Crystal Ball Emoji ]: Projecting Network Anxieties with Alternative Design Metaphors." In DIS '17 Proceedings of the 2017 Conference on Designing Interactive Systems, 1383-93. ACM Press. doi:10.1145/3064663.3064795.

Realpe-Muñoz, Paulo, Cesar A Collazos, Julio Hurtado, Toni Granollers, and Jaime Velasco-Medina. 2016. "An Integration of Usable Security and User Authentication into the ISO 9241-210 and ISO/IEC 25010:2011." In Human Aspects of Information Security, Privacy, and Trust: 4th International Conference, HAS 2016, Held as Part of HCI International 2016, Toronto, ON, Canada, July 17-22, 2016, Proceedings, edited by Theo Tryfonas, 65-76. Cham: Springer International Publishing. doi:10.1007/978-3-319-39381-0_7.

Schön, Donald A. 1979. "Generative Metaphor: A Perspective on Problem-Setting in Social Policy." In Metaphor and Thought, edited by Andrew Ortony, 137-63. Cambridge: Cambridge University Press. doi:10.1017/CBO9781139173865.011. 
Steen, Marc. 2011. "Tensions in Human-Centred Design.” CoDesign 7 (1): 45-60. doi:10.1080/15710882.2011.563314.

Suchman, Lucy. 2007. Human-machine reconfigurations: Plans and situated actions. Cambridge University Press.

Schweikardt, Eric. 2009. "User Centered Is off Center." Interactions 16 (3): 12-15. doi:10.1145/1516016.1516019.

Wakkary, Ron, Doenja Oogjes, Sabrina Hauser, Henry Lin, Cheng Cao, Leo Ma, and Tijs Duel. 2017. "Morse Things." In Proceedings of the 2017 Conference on Designing Interactive Systems - DIS '17, 503-14. New York, New York, USA: ACM Press. doi:10.1145/3064663.3064734.

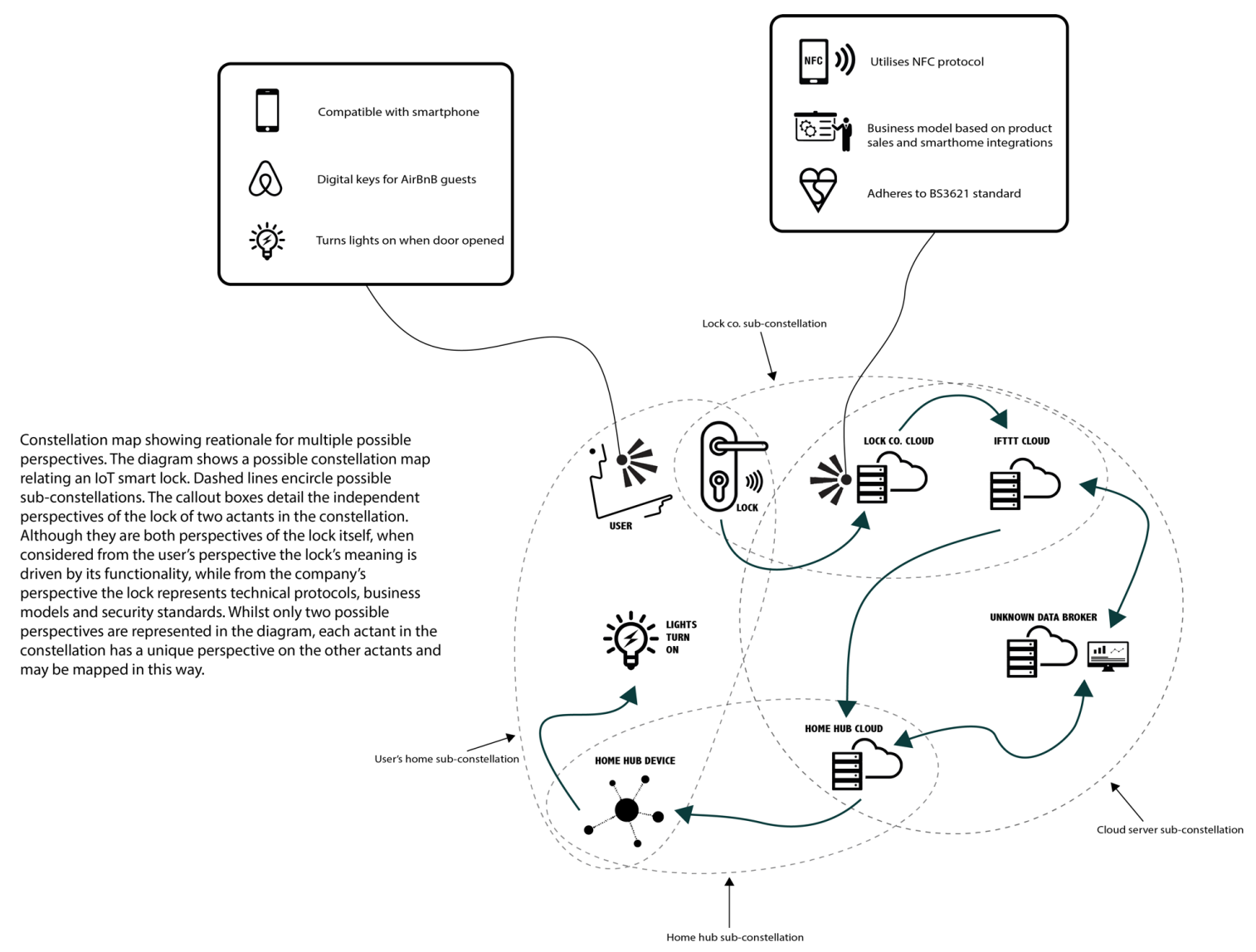

Figure 1. Data flow and constellations for an IoT smart lock. 
Data held locally, with known providers, and with unknown 3 rd parties is represented by the three circles. The lower the chance of identifiability at any given level, the less blurry the edge will be shown. In the example on the right the user is definitely identifiable with the local data whereas in the example on the left the user is almost certainly not idenifiable with the data held by unknown 3rd parties.

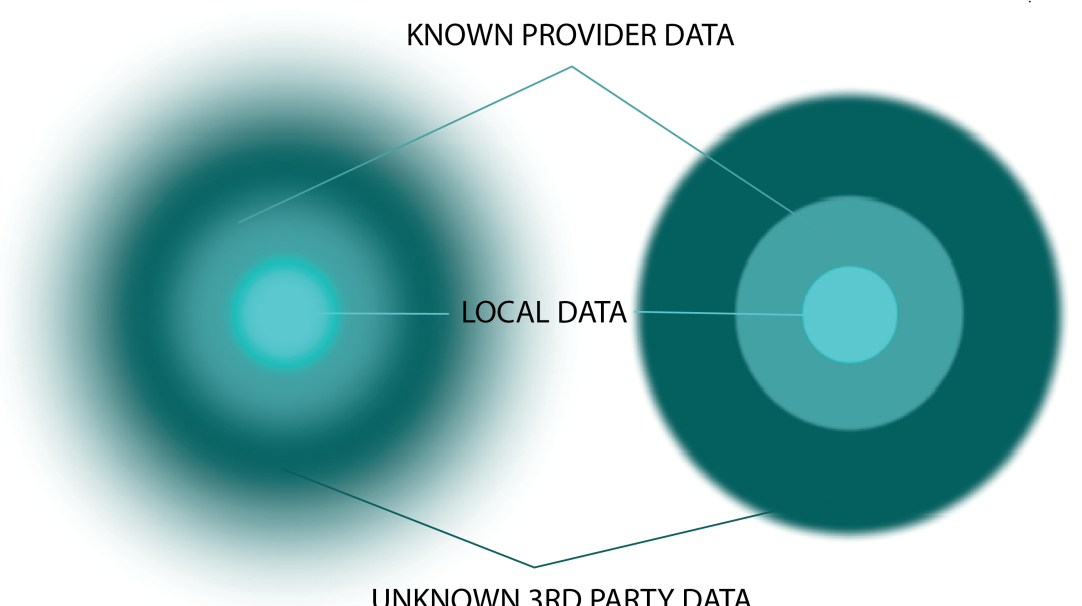

UNKNOWN 3RD PARTY DATA

Figure 2. Concept design for data 'Orbits'.

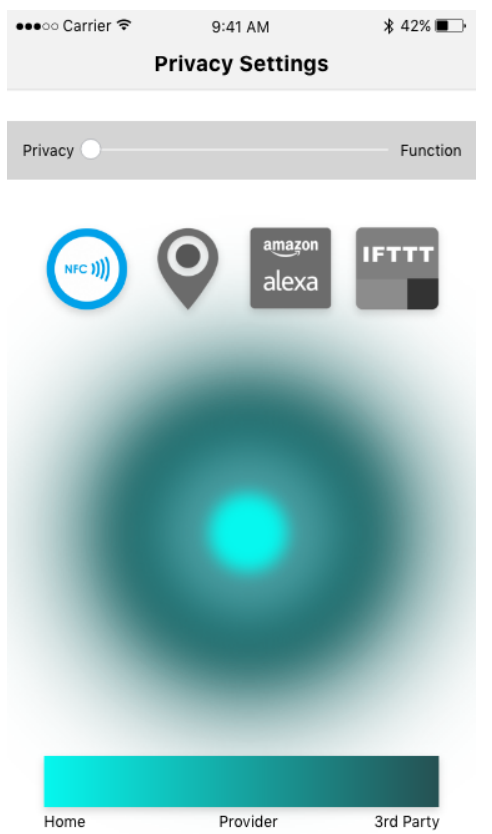

\begin{tabular}{lcc}
... Carrier ₹ & $\begin{array}{c}\text { 9:41 AM } \\
\text { Privacy Settings }\end{array}$ & $* 42 \%$ \\
\hline Privacy & & Function \\
\hline
\end{tabular}
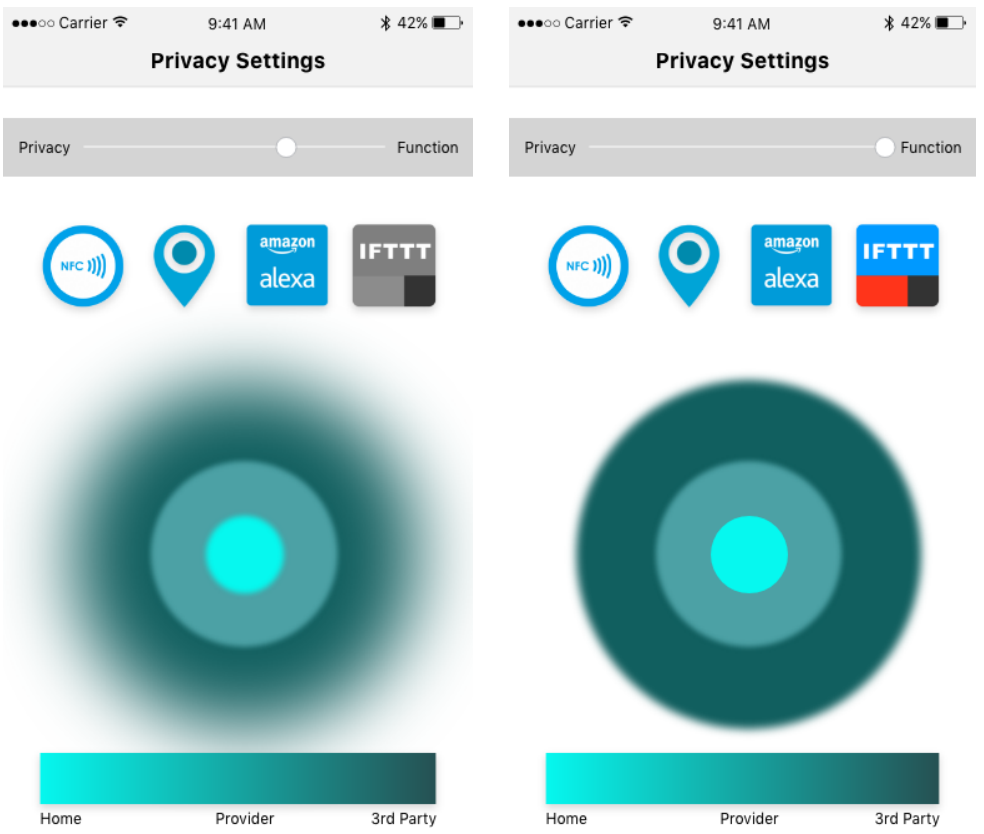

Figure 3. Functionality effecting identifiability as shown on data 'Orbits'. 